\title{
Operator Inequalities of Morrey Spaces Associated with Karamata Regular Variation
}

\author{
Jiajia Wang, ${ }^{1,2}$ Zunwei Fu, ${ }^{2}$ Shaoguang Shi, ${ }^{2}$ and Ling $\mathrm{Mi}^{2}$ \\ ${ }^{1}$ School of Mathematics and Statistics, Shandong Normal University, Jinan 250014, China \\ ${ }^{2}$ Department of Mathematics, Linyi University, Linyi 276005, China
}

Correspondence should be addressed to Shaoguang Shi; shishaoguang@mail.bnu.edu.cn

Received 12 May 2017; Accepted 31 July 2017; Published 24 August 2017

Academic Editor: Guozhen Lu

Copyright (c) 2017 Jiajia Wang et al. This is an open access article distributed under the Creative Commons Attribution License, which permits unrestricted use, distribution, and reproduction in any medium, provided the original work is properly cited.

Karamata regular variation is a basic tool in stochastic process and the boundary blow-up problems for partial differential equations (PDEs). Morrey space is closely related to study of the regularity of solutions to elliptic PDEs. The aim of this paper is trying to bring together these two areas and this paper is intended as an attempt at motivating some further research on these areas. A version of Morrey space associated with Karamata regular variation is introduced. As application, some estimates of operators, especially one-sided operators, on these spaces are considered.

\section{Introduction}

A positive measurable function $w: \mathbb{R} \times \mathbb{R}^{+} \rightarrow \mathbb{R}^{+}$is called regularly varying at infinity with index $\rho$, written as $w \in R V_{\rho}$, if, for each $\xi>0, x_{0} \in \mathbb{R}$ and some $\rho \in \mathbb{R}$,

$$
\lim _{h \rightarrow \infty} \frac{w\left(x_{0}, \xi h\right)}{w\left(x_{0}, h\right)}=\xi^{\rho}
$$

where $I\left(x_{0}, h\right)$ is an interval whose center at $x_{0}$ and radius $h$ and $w\left(x_{0}, h\right)=\int_{I\left(x_{0}, h\right)} w(x) d x$. In particular, when $\rho=0, w$ is called slowly varying at infinity. $w$ is the classical Karamata regular variation. Karamata regular variation theory was first introduced and established by Karamata in 1930. It is a basic tool in stochastic process $[1,2]$ and has been applied to study the boundary behavior of solutions to boundary blow-up elliptic problems and singular nonlinear Dirichlet problems; for some of this work, see [3-7] and the references given there.

If $w \in R V_{0}$, then

$$
\lim _{h \rightarrow \infty} \frac{w\left(x_{0}, \xi h\right)}{w\left(x_{0}, h\right)}=1
$$

holds uniformly for $\xi \in\left[C_{1}, C_{2}\right]$ with $0<C_{1}<C_{2}$ [5]. And for $s>0$ and $h \rightarrow \infty$, the following asymptotic behavior is true:

$$
\int_{h}^{\infty} \frac{w\left(x_{0}, t\right)}{t^{s+1}} d t \simeq \frac{w\left(x_{0}, h\right)}{s h^{s+1}}
$$

Karamata regular variation at 0 can also be defined by a positive measurable function $w$ with $h \rightarrow \infty$ replaced by $h \rightarrow 0$.

In [8], Nakai introduced a generalized weighted Morrey apace with the weight function $w$ satisfying the following conditions:

$$
\begin{aligned}
\frac{1}{C} & \leq \frac{w(a, t)}{w(a, h)} \leq C, \quad h \leq t \leq 2 h, \\
\int_{h}^{\infty} \frac{w(a, t)}{t^{2}} d t & \leq C \frac{w(a, h)}{h},
\end{aligned}
$$

where any $a \in \mathbb{R}$. Inspired by Nakai, a general case of (2) and (3) can be defined as

$$
\frac{1}{C} \leq \frac{w\left(x_{0}, \xi h\right)}{w\left(x_{0}, h\right)} \leq C,
$$


where $\xi \in\left[C_{1}, C_{2}\right]$ with $0<C_{1}<C_{2}$ and

$$
\int_{h}^{\infty} \frac{w\left(x_{0}, t\right)}{t^{s+1}} d t \leq C \frac{w\left(x_{0}, h\right)}{h^{s}} .
$$

It is of interest to know that when $h \rightarrow 0$ or $h \rightarrow \infty$ in (6) and (7), the function $w$ can be seen as Karamata regular variation at 0 and infinity, respectively.

Let $w$ satisfy (6) and (7). Then the Morrey space associated with Karamata regular variation (K-Morrey space) can be adopted from [8] as

$$
\begin{aligned}
& M_{K}^{p, w}(\mathbb{R})=\left\{f \in L_{\mathrm{loc}}^{p}(\mathbb{R}):\|f\|_{M_{K}^{p, w}(\mathbb{R})}\right. \\
& \left.\quad=\sup _{I} \frac{1}{w(I)} \int_{I}|f(x)|^{p} d x<\infty\right\}, \quad 1 \leq p<\infty .
\end{aligned}
$$

It is obvious that $M_{K}^{p, w}(\mathbb{R})$ is a Banach space with norm $\|f\|_{M_{K}^{p, w}(\mathbb{R})}$. If $w(I) \equiv 1$, then $M_{K}^{p, w}(\mathbb{R})=L^{p}(\mathbb{R})$. If $w(I)=r$, then $M_{K}^{p, w}(\mathbb{R})=L^{\infty}(\mathbb{R})$. And if $w(I)=r^{\lambda}$ with $0<\lambda<1$, then $M_{K}^{p, w}(\mathbb{R})$ is the classical Morrey space which was first introduced by Morrey [9] to investigate the local behavior of solutions to the second order elliptic PDEs.

It is also worth pointing out that when $s=1, \xi \in[1,2]$, and (6) and (7) are exactly the same as (4) and (5), $M_{K}^{p, w}(\mathbb{R})$ is the generalized Morrey space $L^{p, w}(\mathbb{R})$ introduced by Nakai [8] with $n=1$. In his well-known paper, Nakai proved the interesting result that the Hardy-Littlewood maximal operator, singular integral operator, and the Riesz potential were bounded on certain space. As stated in [8], we can also prove that $w(I)=\left(\int_{I} u(x)\right)^{\alpha}(0<\alpha<1)$ with $1 \leq p \leq 1 / \alpha$ and $u(x) \in A_{p}$ [10] is an example of functions satisfying (6) and (7).

In this paper, we shall consider some estimates for onesided operators on the $K$-Morrey space $M_{K}^{p, w}(\mathbb{R})$. Let us first recall some basic definitions of one-sided operators. The reasons to study one-sided operators involve the requirements of ergodic theory [11]. The study of weighted theory for onesided operators was first introduced by Sawyer [12] and many authors thereafter ([13-19]). The one-sided Hardy-Littlewood maximal operators [12] are defined by

$$
\begin{aligned}
& M^{+} f(x)=\sup _{h>0} \frac{1}{h} \int_{x}^{x+h}|f(y)| d y, \\
& M^{-} f(x)=\sup _{h>0} \frac{1}{h} \int_{x-h}^{x}|f(y)| d y,
\end{aligned}
$$

which arise in the ergodic maximal function. It is well known that $M^{+}$and $M^{-}$are bounded on $L^{p}(\mathbb{R})$ spaces $(1<p<\infty)$ and bounded from $L^{1}(\mathbb{R})$ spaces to weak $L^{1}(\mathbb{R})$ spaces. Such operators are also bounded on $K$-Morrey spaces, which we now formulated as follows.

Theorem 1. Let $w$ satisfy (6) and (7) with $0<s \leq 1$. Then one has the following:

(a) For $1<p<\infty$, there is a constant $C>0$ such that

$$
\left\|M^{+} f\right\|_{M_{K}^{p, w}(\mathbb{R})} \leq C\|f\|_{M_{K}^{p, w}(\mathbb{R})} .
$$

(b) There is a constant $C>0$ such that, for any $t>0$ and for any $I \subset \mathbb{R}$, one has

$$
\left|\left\{x \in I: M^{+} f(x)>t\right\}\right| \leq C \frac{w(I)}{t}\|f\|_{M_{K}^{1, w}(\mathbb{R})} .
$$

Let $T^{+}$be an one-sided integral operator with one-sided kernel $K^{+}(x, y)$ supported in $\mathbb{R}^{-}=(-\infty, 0)$ and satisfy

$$
\left|K^{+}(x, y)\right| \leq \frac{C}{(y-x)} .
$$

That is,

$$
\begin{aligned}
& T^{+} f(x)=\text { p.v. } \int_{x}^{\infty} K^{+}(x, y) f(y) d y \\
& \\
& x \in(\operatorname{supp} f)^{c} .
\end{aligned}
$$

Both the one-sided Calderón-Zygmund singular integral operator [13] and the one-sided oscillatory singular operator [17] are examples of operators $T^{+}$. Our second result is as follows.

Theorem 2. Let $w$ satisfy (6) and (7) with $0<s \leq 1$. Then one has the following:

(a) If $T^{+}$is bounded on $L^{p}(\mathbb{R})$ with $1<p<\infty$, then there is a constant $C>0$ such that

$$
\left\|T^{+} f\right\|_{M_{K}^{p, w}(\mathbb{R})} \leq C\|f\|_{M_{K}^{p, w}(\mathbb{R})} .
$$

(b) If $T^{+}$is bounded from $L^{1}(\mathbb{R})$ space to weak $L^{1}(\mathbb{R})$ space, then there is a constant $C>0$ such that for any $t>0$ and for any $I$

$$
\left|\left\{x \in I: T^{+} f(x)>t\right\}\right| \leq C \frac{w(I)}{t}\|f\|_{M_{K}^{1, w}(\mathbb{R})} .
$$

Remark 3. Theorem 2 provides a criterion for the boundedness of one-sided singular integral operators on $K$-Morrey spaces.

By the corresponding boundedness in $[13,15,17]$, both the one-sided Calderón-Zygmund singular integral operator and the one-sided oscillatory singular operator satisfy Theorem 2 .

In the fractional case, both the Rieman-Liouville fractional integral

$$
I_{\alpha}^{+} f(x)=\int_{x}^{\infty} \frac{f(y)}{(y-x)^{1-\alpha}} d y, \quad 0<\alpha<1,
$$

and the Weyl fractional integral

$$
I_{\alpha}^{-} f(x)=\int_{-\infty}^{x} \frac{f(y)}{(x-y)^{1-\alpha}} d y, \quad 0<\alpha<1,
$$

are examples of one-sided fractional integrals. Without loss of generality, we take $I_{\alpha}^{+}$as our model in the following analysis. The last goal of this section is to show that $I_{\alpha}^{+}$is also bounded on $K$-Morrey space, which can be stated in the following theorem. 
Theorem 4. Let $0<\alpha<1,0<s \leq 1,1 \leq p<s / \alpha$, $1 / q=1 / p-\alpha, w$ satisfy (6), and $\int_{h}^{\infty}\left(w\left(x_{0}, t\right) / t^{s-\alpha p+1}\right) d t \leq$ $C\left(w\left(x_{0}, h\right) / h^{s-\alpha p}\right)$ with $x_{0} \in \mathbb{R}$. Then one has the following:

(a) If $1<p<\infty$, then there is a constant $C>0$ such that

$$
\left\|I_{\alpha}^{+} f\right\|_{M_{K}^{q, q w / p}(\mathbb{R})} \leq C\|f\|_{M_{K}^{p, w}(\mathbb{R})} .
$$

(b) If $p=1$, then there is a constant $C>0$ such that for any $t>0$ and for any $I$

$$
\left|\left\{x \in I: I_{\alpha}^{+} f(x)>t\right\}\right| \leq C \frac{w(I)}{t^{q}}\|f\|_{M_{K}^{1, w}(\mathbb{R})}^{q} .
$$

Section 2 contains the proofs of Theorems 1-4. In Section 3 , we extend the main results to $n$-dimensional case, which cover the main results of [8]. Throughout this paper, $C$ is a constant which may change from line to line.

\section{Preliminaries}

In this section, some lemmas are described by some methods adopted from [20].

Lemma 5 (see [21]). Let $\varphi \geq 0$ be measurable functions. Then one has the following: that

(a) For every $1<p<\infty$, there is a constant $C>0$ such

$$
\int_{\mathbb{R}}\left(M^{+} f(x)\right)^{p} \varphi(x) d x \leq C \int_{\mathbb{R}}|f(x)|^{p} M^{-} \varphi(x) d x .
$$

(b) There is a constant $C>0$ such that

$$
\int_{\left\{x: M^{+} f(x)>t\right\}} \varphi(x) d x \leq \frac{C}{t} \int_{\mathbb{R}}|f(x)| M^{-} \varphi(x) d x .
$$

The principal significance of Lemma 5 is that it allows one to obtain a version of one-sided Fefferman-Stein inequality. The following lemma will prove extremely useful in the proofs of the main results.

Lemma 6. Let $0<\delta \leq 1,0<s \leq 1, w$ satisfy (6), and

$$
\int_{h}^{\infty} \frac{w\left(x_{0}, t\right)}{t^{s \delta+1}} d t \leq C \frac{w\left(x_{0}, h\right)}{h^{s \delta}} .
$$

Then for $1 \leq p<\infty$, there is a constant $C>0$ such that

$$
\int|f(x)|^{p}\left(M^{-} \chi_{I}(x)\right)^{\delta} d x \leq C w(I)\|f\|_{M_{K}^{p, w}(\mathbb{R})}^{p} .
$$

Proof. The proof of Lemma 6 has a root in [8, Lemma 1]. We adopted its proof here for the one-sided case. Let $\chi_{I}$ be the characteristic function of $I=I\left(x_{0}, h\right)$. Then $M^{-} \chi_{I} \leq 1$ for $x \in 2 I$. For $x \in 2^{k+1} I / 2^{k} I$ and $y \in I$, the following can be shown:

$$
\begin{aligned}
& M^{-} \chi_{I}(x)= \sup _{h>0} \frac{1}{2^{k} h} \int_{x-2^{k} h}^{x} \chi_{I}(y) d y \\
&=\sup _{h>0} \frac{1}{2^{k} h} \int_{x_{0}}^{x_{0}+h} \chi_{I}(y) d y \leq C 2^{-k}, \\
& k=1,2,3, \ldots .
\end{aligned}
$$

This clearly forces

$$
\begin{aligned}
& \int|f(x)|^{p}\left(M^{-} \chi_{I}(x)\right)^{\delta} d x \\
& \quad \leq C\left\{\int_{2 I}|f(x)|^{p}\left(M^{-} \chi_{I}(x)\right)^{\delta} d x\right. \\
& \left.+\sum_{k=1}^{\infty} \int_{2^{k+1} I 2^{k} I}|f(x)|^{p}\left(M^{-} \chi_{I}(x)\right)^{\delta} d x\right\} \\
& \leq C\left\{\int_{2 I}|f(x)|^{p} d x\right. \\
& \left.+\sum_{k=1}^{\infty} \int_{2^{k+1} I / 2^{k} I}|f(x)|^{p} 2^{-k \delta} d x\right\} \leq C\{w(2 I) \\
& \left.+\sum_{k=1}^{\infty} 2^{-k \delta} w\left(2^{k+1} I\right)\right\}\|f\|_{M_{K}^{p, w}(\mathbb{R})}^{p} \\
& \quad \leq C h^{\delta} \sum_{k=0}^{\infty} \frac{w\left(2^{k} I\right)}{\left(2^{k} h\right)^{\delta}}\|f\|_{M_{K}^{p, w}(\mathbb{R})}^{p} .
\end{aligned}
$$

We conclude from (6) that

$$
\frac{1}{C} \leq \frac{w\left(x_{0}, t\right)}{w\left(x_{0}, 2^{k} h\right)} \leq C, \quad 2^{k} h \leq t \leq 2^{k+1} h,
$$

hence that

$$
\begin{aligned}
\int_{2^{k} h}^{2^{k+1} h} \frac{w\left(x_{0}, t\right)}{t^{s \delta+1}} d t & \geq \frac{1}{C} \int_{2^{k} h}^{2^{k+1} h} \frac{w\left(x_{0}, 2^{k} h\right)}{t^{s \delta+1}} d t \\
& \geq \frac{1}{C} \frac{w\left(x_{0}, 2^{k} h\right)}{\left(2^{k} h\right)^{s \delta}},
\end{aligned}
$$

and finally that

$$
\int_{2^{k} h}^{2^{k+1} h} \frac{w\left(x_{0}, t\right)}{t^{s \delta+1}} d t \leq C \frac{w\left(x_{0}, 2^{k} h\right)}{\left(2^{k} h\right)^{s \delta}} .
$$

Equations (27) and (28) show that $w\left(x_{0}, 2^{k} h\right) /\left(2^{k} h\right)^{s \delta}$ is comparable to $\int_{2^{k} h}^{2^{k+1} h}\left(w\left(x_{0}, t\right) / t^{s \delta+1}\right) d t$, which derives that

$$
\begin{aligned}
& \int|f(x)|^{p}\left(M^{-} \chi_{I}(x)\right)^{\delta} d x \\
& \leq C h^{\delta} \sum_{k=0}^{\infty} \frac{\left(\left(2^{k} h\right)^{s \delta}\right)}{\left(2^{k} h\right)^{\delta}} \frac{w\left(2^{k} I\right)}{\left(2^{k} h\right)^{s \delta}}\|f\|_{M_{K}^{p, w}(\mathbb{R})}^{p} \\
& \leq C h^{\delta} \sum_{k=0}^{\infty}\left(\left(2^{k} h\right)^{(s-1) \delta}\right) \int_{2^{k} h}^{2^{k+1} h} \frac{w\left(x_{0}, t\right)}{t^{s \delta+1}} d t\|f\|_{M_{K}^{p, w}(\mathbb{R})}^{p} \\
& \leq C h^{s \delta} \sum_{k=0}^{\infty} \int_{2^{k} h}^{2^{k+1} h} \frac{w\left(x_{0}, t\right)}{t^{s \delta+1}} d t\|f\|_{M_{K}^{p, w}(\mathbb{R})}^{p}
\end{aligned}
$$




$$
\begin{aligned}
& \leq C h^{s \delta} \int_{h}^{\infty} \frac{w\left(x_{0}, t\right)}{t^{s \delta+1}} d t\|f\|_{M_{K}^{p, w}(\mathbb{R})}^{p} \\
& \leq C w\left(x_{0}, h\right)\|f\|_{M_{K}^{p, w}(\mathbb{R})}^{p} .
\end{aligned}
$$

On account of the estimates given above, Lemma 6 is proved.

Lemma 7 (see [8]). Suppose that $\varphi(h): \mathbb{R}^{+} \rightarrow \mathbb{R}^{+}$. If there is a constant $C>0$ such that, for any $h>0, \int_{h}^{\infty}(\varphi(t) / t) d t \leq$ $C \varphi(h)$, then there are constants $\varepsilon>0$ and $C^{\prime}>0$ such that, for any $h>0$,

$$
\int_{h}^{\infty} \frac{\varphi(t) t^{\varepsilon}}{t} d t \leq C^{\prime} \varphi(h) h^{\varepsilon} .
$$

The remainder lemma of this section will be devoted to the boundedness of one-sided fractional integrals on Lebesgue spaces.

Lemma 8 (see [14]). Let $0<\alpha<1,1 \leq p<s / \alpha, 0<s \leq 1$, and $1 / q=1 / p-\alpha$. Then there exists constant $C>0$ such that

$$
\begin{aligned}
\left\|I_{\alpha}^{+} f(x)\right\|_{L^{q}(\mathbb{R})} & \leq C\|f\|_{L^{p}(\mathbb{R})}, \quad p>1 ; \\
\left\|I_{\alpha}^{+} f(x)\right\|_{L^{q, \infty}(\mathbb{R})} & \leq C\|f\|_{L^{1}(\mathbb{R})} .
\end{aligned}
$$

Having disposed of the above lemmas, the estimates for the one-sided operators on $K$-Morrey spaces can be proved in this section. The method used here was partly adopted from [8].

Proof of Theorem 1. Let us first prove (a). Taking into account (20) and Lemma 6 with $\delta=1$, the following can be shown easily

$$
\begin{aligned}
& \int_{I}\left(M^{+} f(x)\right)^{p} \chi_{I}(x) d x \leq C \int|f(x)|^{p} M^{-} \chi_{I}(x) d x \\
& \quad \leq C w(I)\|f\|_{M_{K}^{p, w}(\mathbb{R})}^{p}
\end{aligned}
$$

Applying (21) and Lemma 6 with $\delta=1$, we note that

$$
\begin{aligned}
\mid\{x & \left.\in I: M^{+} f(x)>t\right\} \mid=\int_{\left\{x: M^{+} f(x)>t\right\}} \chi_{I}(x) d x \\
& \leq \frac{C}{t} \int|f(x)| M^{-} \chi_{I}(x) d x \\
& \leq\left(\frac{C}{t}\right) w(I)\|f\|_{M_{K}^{1, w}(\mathbb{R})} .
\end{aligned}
$$

The proof of Theorem 1 is completed.

Proof of Theorem 2. (a) For any $I$, let $f=f_{2 I}+f_{(2 I)^{c}}=: f_{1}+f_{2}$ to produce

$$
T^{+} f(x) \leq T^{+} f_{1}(x)+T^{+} f_{2}(x)
$$

We conclude from the fact that $T^{+}$is bounded on $L^{p}\left(\mathbb{R}^{n}\right)$ that

$$
\int_{I}\left|T^{+} f_{1}(x)\right|^{p} d x \leq\left\|T^{+} f_{1}\right\|_{L^{p}}^{p} \leq C w(2 I)\|f\|_{M_{K}^{p, w}(\mathbb{R})}^{p} .
$$

The task is now to deal with the term $T^{+} f_{2}$. The fact that $x \in I$ and $y \in(2 I)^{c}$ allows the user to estimate $M^{-} \chi_{I}$ as

$$
M^{-} \chi_{I}(y)=\sup _{h>0} \frac{1}{h} \int_{y-h}^{y} \chi_{I}(z) d z \geq \frac{h}{y-x} .
$$

This clearly forces

$$
\begin{aligned}
& \int\left|K^{+}(x, y) f_{2}(y)\right| d y \leq C \int_{(2 I)^{c}} \frac{\left|f_{2}(y)\right|}{y-x} d y \\
& \leq \frac{C}{h} \int_{(2 I)^{c}}|f(y)| M^{-} \chi_{I}(y) d y .
\end{aligned}
$$

Applying Lemma 7 to $\varphi(t)=w\left(x_{0}, t\right) / t^{s}$, it is easy to check that

$$
\int_{h}^{\infty} \frac{w\left(x_{0}, t\right)}{t^{s-\varepsilon+1}} d t \leq C \frac{w\left(x_{0}, h\right)}{h^{s-\varepsilon}} .
$$

Let $\delta=(s-\varepsilon) / s$. Using Hölder's inequality, it may be concluded that

$$
\begin{aligned}
& \int\left|K^{+}(x, y) f_{2}(y)\right| d y \\
& \leq C\left(\frac{1}{h} \int|f|^{p}\left(M^{-} \chi_{I}(y)\right)^{\delta} d y\right)^{1 / p} \\
& \cdot\left(\frac{1}{h} \int\left(M^{-} \chi_{I}(y)\right)^{(p-\delta) /(p-1)} d y\right)^{(p-1) / p}=: J_{1} \times J_{2} .
\end{aligned}
$$

Repeated application of Lemma 6 enables us to write

$$
J_{1} \leq C h^{-1 / p} w(I)^{1 / p}\|f\|_{M_{K}^{p, w}(\mathbb{R})} .
$$

For the term $J_{2}$, the fact that $M^{-} \chi_{I}(y) \leq 1, y \in 2 I$, and $M^{-} \chi_{I}(y) \leq 2^{-k}$ for $y \in\left(2^{k+1} I\right) /\left(2^{k} I\right)$ shows that

$$
\begin{aligned}
J_{2} \leq & \frac{1}{h}\left(\int_{2 I} d y\right. \\
& \left.+\sum_{k=1}^{\infty} \int_{2^{k+1} I / 2^{k} I}\left(M^{-} \chi_{I}(y)\right)^{(p-\delta) /(p-1)} d y\right) \leq \frac{C}{h} \\
& \cdot \sum_{k=0}^{\infty} 2^{-k(p-\delta) /(p-1)}\left|2^{k+1} I\right| \leq C \sum_{k=0}^{\infty} 2^{-k \varepsilon /(p-1)} \leq C .
\end{aligned}
$$

Hence,

$$
\int\left|K^{+}(x, y) f_{2}(y)\right| d y \leq C h^{-1 / p} w(I)^{1 / p}\|f\|_{M_{K}^{p, w}(\mathbb{R})} .
$$

The proof of (a) is completed by showing that

$$
\begin{aligned}
\int_{I}\left|T^{+} f_{2}(x)\right|^{p} d x & \leq C \int_{I} \frac{1}{h} w(I)\|f\|_{M_{K}^{p, w}(\mathbb{R})}^{p} d x \\
& \leq C w(I)\|f\|_{M_{K}^{p, w}(\mathbb{R})}^{p} .
\end{aligned}
$$


(b) For $f$ and $I$, let $f=f_{2 I}+f_{(2 I)^{c}}=: f_{1}+f_{2}$ to produce

$$
T^{+} f(x) \leq T^{+} f_{1}(x)+T^{+} f_{2}(x) .
$$

Since $T^{+}$is bounded from $L^{1}$ to $L^{1, \infty}$, the following can be proved:

$$
\left|\left\{x \in I: T^{+} f_{1}(x)>t\right\}\right| \leq \frac{C}{t} w(I)\|f\|_{M_{K}^{1, w}(\mathbb{R})} .
$$

Applying the same analysis as (a) and Lemma 6 with $p=\delta=$ 1 ,

$$
\begin{aligned}
\left|T^{+} f_{2}(x)\right| & \leq \frac{C}{h} \int|f(y)| M^{-} \chi_{I}(y) d y \\
& \leq \frac{C}{h} w(I)\|f\|_{M_{K}^{1, w}(\mathbb{R})} .
\end{aligned}
$$

Therefore,

$$
\left|\left\{x \in I: T^{+} f_{2}(x)>t\right\}\right| \leq \frac{C}{t} w(2 I)\|f\|_{M_{K}^{1, w}(\mathbb{R})} .
$$

We have thus completed the proof of Theorem 2.

Proof of Theorem 4. An argument similar to that of Theorem 2 can be used to prove Theorem 4. (a) For any $I$, let $f=f_{2 I}+$ $f_{(2 I)^{c}}=: f_{1}+f_{2}$ to produce

$$
I_{\alpha}^{+} f(x) \leq I_{\alpha}^{+} f_{1}(x)+I_{\alpha}^{+} f_{2}(x) .
$$

Applying Lemma 8, we conclude that

$$
\int_{I}\left|I_{\alpha}^{+} f_{1}(x)\right|^{q} d x \leq\left\|I_{\alpha}^{+} f_{1}\right\|_{L^{q}}^{q} \leq C\left\|f_{1}\right\|_{L^{p}(\mathbb{R})}^{q},
$$

which implies

$$
\left(\frac{1}{w(I)^{q / p}} \int_{I}\left|I_{\alpha}^{+} f_{1}(x)\right|^{q} d x\right)^{1 / q} \leq C\|f\|_{M_{K}^{p, w}(\mathbb{R})} .
$$

For $x \in I$ and $y \in(2 I)^{c}$, the argument in (36) shows that

$$
\frac{1}{(y-x)^{1-\alpha}} \leq C\left(\frac{M^{-} \chi_{I}(y)}{|I|}\right)^{1-\alpha} .
$$

Hence,

$$
\begin{aligned}
\left|I_{\alpha}^{+} f_{2}(x)\right| & \leq C \int_{(2 I)^{c}} \frac{f_{2}(y)}{(y-x)^{1-\alpha}} d y \\
& \leq \frac{C}{h^{1-\alpha}} \int_{(2 I)^{c}}|f(y)|\left(M^{-} \chi_{I}(y)\right)^{1-\alpha} d y .
\end{aligned}
$$

Applying Lemma 7 to $\varphi(t)=w\left(x_{0}, t\right) / t^{s-\alpha p}$, the following is true:

$$
\int_{h}^{\infty} \frac{w\left(x_{0}, t\right)}{t^{s-\alpha p-\varepsilon+1}} d t \leq C \frac{w\left(x_{0}, h\right)}{h^{s-\alpha p-\varepsilon}} .
$$

Let $\delta=(s-\alpha p-\varepsilon) / s$. Hölder's inequality can be used to obtain

$$
\begin{gathered}
\left|I_{\alpha}^{+} f_{2}(x)\right| \leq C\left(\frac{1}{h^{1-\alpha}} \int|f|^{p}\left(M^{-} \chi_{I}(y)\right)^{\delta} d y\right)^{1 / p} \\
\cdot\left(\frac{1}{h} \int\left(M^{-} \chi_{I}(y)\right)^{(p-\alpha p-\delta) /(p-1)} d y\right)^{(p-1) / p} .
\end{gathered}
$$

The fact

$$
\begin{aligned}
& \frac{1}{h} \int\left(M^{-} \chi_{I}(y)\right)^{(p-\alpha p-\delta) /(p-1)} d y \leq \frac{1}{h}\left(\int_{2 I} d y\right. \\
& \left.+\sum_{k=1}^{\infty} \int_{2^{k+1} I / 2^{k} I}\left(M^{-} \chi_{I}(y)\right)^{(p-\alpha p-\delta) /(p-1)} d y\right) \leq \frac{C}{h} \\
& \quad \cdot \sum_{k=1}^{\infty} 2^{-k(p-\alpha p-\delta) /(p-1)}\left|2^{k+1} I\right| \leq C
\end{aligned}
$$

and Lemma 6 with $\delta=1-\alpha p / s<1$ allow us to estimate $I_{\alpha}^{+} f_{2}(x)$ as

$$
\left|I_{\alpha}^{+} f_{2}(x)\right| \leq C h^{-1 / q} w(I)^{1 / p}\|f\|_{M_{K}^{p, w}(\mathbb{R})},
$$

which produces

$$
\left(\frac{1}{w(I)^{q / p}} \int_{I}\left|I_{\alpha}^{+} f_{2}(x)\right|^{q} d x\right)^{1 / q} \leq C\|f\|_{M_{K}^{p, w}(\mathbb{R})} .
$$

(b) For $f$ and $I$, let $f=f_{2 I}+f_{(2 I)^{c}}=: f_{1}+f_{2}$ produce

$$
I_{\alpha}^{+} f(x) \leq I_{\alpha}^{+} f_{1}(x)+I_{\alpha}^{+} f_{2}(x) .
$$

The fact that $I_{\alpha}^{+}$is bounded from $L^{1}$ to $L^{q, \infty}$ allows us to get

$$
\left|\left\{x \in I: I_{\alpha}^{+} f_{1}(x)>t\right\}\right| \leq \frac{C}{t^{q}}\left(w(2 I)\|f\|_{M_{K}^{1, w}(\mathbb{R})}\right)^{q} .
$$

Applying the same analysis as that of (a) and Lemma 6 with $p=1, \delta=1-\alpha=1 / q<1$, the following can be confirmed easily:

$$
\left|I_{\alpha}^{+} f_{2}(x)\right| \leq \frac{C}{h^{1 / q}} w(I)\|f\|_{M_{K}^{1, w}(\mathbb{R})} .
$$

This produces the following inequality:

$$
\left|\left\{x \in I: I_{\alpha}^{+} f_{2}(x)>t\right\}\right| \leq \frac{C}{t^{q}}\left(w(I)\|f\|_{M_{K}^{1, w}(\mathbb{R})}\right)^{q},
$$

which is our desired result.

\section{Boundedness of Operators on $n$-Dimensional $K$-Morrey Spaces}

Since one-sided operators are defined on $\mathbb{R}$, we built Theorems 1-4 in one dimension. The theorems in Section 2 gain interest if we realize that they are still hold for $n$-dimension. In fact, we can also define $K$-Morrey space on $\mathbb{R}^{n}(n \geq 2)$ 
with $0<s \leq n$ and consider the boundedness of HardyLittlewood maximal operator, singular integral operator, and the Riesz potential on these spaces applying the method in [8] with only a slight modification. Let $w: \mathbb{R}^{n} \times \mathbb{R}^{+} \rightarrow \mathbb{R}^{+}$and $\Omega\left(x_{0}, r\right)$ be the cube whose center at $x_{0}$ with edges has length $r$ and is parallel to the coordinate axes. Then the definition of $n$ dimensional Morrey space associated with Karamata regular variation ( $K$-Morrey space) can be defined by

$$
\begin{aligned}
& M_{K}^{p, w}\left(\mathbb{R}^{n}\right)=\left\{f \in L_{\mathrm{loc}}^{p}\left(\mathbb{R}^{n}\right):\|f\|_{M_{K}^{p, w}\left(\mathbb{R}^{n}\right)}\right. \\
& \left.=: \sup _{B \subset \mathbb{R}^{n}} \frac{1}{w(B)} \int_{B}|f(x)|^{p} d x<\infty\right\}, \quad 1 \leq p<\infty,
\end{aligned}
$$

if $w$ satisfies the following conditions:

$$
\frac{1}{C} \leq \frac{w\left(x_{0}, \xi r\right)}{w\left(x_{0}, r\right)} \leq C,
$$

where $\xi \in\left[C_{1}, C_{2}\right]$ with $0<C_{1}<C_{2}$ and

$$
\int_{r}^{\infty} \frac{w\left(x_{0}, t\right)}{t^{s+1}} d t \leq C \frac{w\left(x_{0}, r\right)}{h^{s}}, \quad 0<s \leq n .
$$

Theorem 9. Let $M$ be the Hardy-Littlewood maximal operator

$$
M f(x)=\sup _{\Omega \ni x} \frac{1}{|\Omega|} \int_{\Omega}|f(y)| d y .
$$

Assume that $w$ satisfies (63) and (64) with $0<s \leq n$. Then one has the following:

(a) For $1<p<\infty$, there is a constant $C>0$ such that

$$
\|M f\|_{M_{K}^{p, w}\left(\mathbb{R}^{n}\right)} \leq C\|f\|_{M_{K}^{p, w}\left(\mathbb{R}^{n}\right)} .
$$

(b) There is a constant $C>0$ such that for any $\lambda>0$ and for any cube $\Omega$

$$
|\{x \in \Omega: M f(x)>\lambda\}| \leq C \frac{w(\Omega)}{t}\|f\|_{M_{K}^{1, w}\left(\mathbb{R}^{n}\right)} .
$$

Theorem 10. Let $T$ be a singular integral operator

$$
T f(x)=p . v . \int_{\mathbb{R}^{n}} K(x, y) f(y) d y, \quad x \in(\operatorname{supp} f)^{c},
$$

with the kernel $K(x, y)$ satisfying

$$
|K(x, y)| \leq \frac{C}{|x-y|^{n}}
$$

Assume that $w$ satisfies (63) and (64) with $0<s \leq n$. Then one has the following:

(a) If $T$ is bounded on $L^{p}\left(\mathbb{R}^{n}\right)$ with $1<p<\infty$, then there is a constant $C>0$ such that

$$
\|T f\|_{M_{K}^{p, w}\left(\mathbb{R}^{n}\right)} \leq C\|f\|_{M_{K}^{p, w}\left(\mathbb{R}^{n}\right)} .
$$

(b) If $T$ is bounded from $L^{1}\left(\mathbb{R}^{n}\right)$ space to weak $L^{1}\left(\mathbb{R}^{n}\right)$ space, then there is a constant $C>0$ such that, for any $\lambda>0$ and for any cube $\Omega \subset \mathbb{R}^{n}$, one has

$$
|\{x \in \Omega: T f(x)>\lambda\}| \leq C \frac{w(\Omega)}{\lambda}\|f\|_{M_{K}^{1, w}\left(\mathbb{R}^{n}\right)} .
$$

The last result is about the Riesz potential $I_{\alpha}$

$$
I_{\alpha} f(x)=\int_{\mathbb{R}^{n}} \frac{f(y)}{|y-x|^{n-\alpha}} d y, \quad 0<\alpha<n .
$$

Theorem 11. Let $0<\alpha<n, 0<s \leq n, 1 \leq p<s / \alpha$, $1 / q=1 / p-\alpha / n, w$ satisfy $(63)$, and $\int_{r}^{\infty}\left(w\left(x_{0}, t\right) / t^{s-\alpha p+1}\right) d t \leq$ $C\left(w\left(x_{0}, r\right) / r^{s-\alpha p}\right)$ with $x_{0} \in \mathbb{R}^{n}$. Then one has the following:

(a) If $1<p<\infty$, then there is a constant $C>0$ such that

$$
\left\|I_{\alpha} f\right\|_{M_{K}^{q, q w / p}\left(\mathbb{R}^{n}\right)} \leq C\|f\|_{M_{K}^{p, w}\left(\mathbb{R}^{n}\right)} .
$$

(b) If $p=1$, then there is a constant $C>0$ such that for any $\lambda>0$ and for any cube $\Omega \subset \mathbb{R}^{n}$

$$
\left|\left\{x \in \Omega: I_{\alpha} f(x)>\lambda\right\}\right| \leq C \frac{w(\Omega)}{\lambda^{q}}\|f\|_{M_{K}^{1, w}\left(\mathbb{R}^{n}\right)}^{q} .
$$

When $s=n$, it is of interest to know that Theorems 9-11 can be seen as an extension of that of [8] in the sense that these theorems agree with [8, Theorems 1-3], respectively.

With a slight modification in the proofs of $[8$, Theorems 1-3], Theorems 9-11 can be obtained easily; we omit its proof here for the similarity.

\section{Conflicts of Interest}

The authors declare that they have no conflicts of interest.

\section{Authors' Contributions}

All authors read and approved the final manuscript.

\section{Acknowledgments}

This work was partially supported by National Natural Science Foundation of China (Grant nos. 11671185, 11771195, and 11771196), the key Laboratory of Complex Systems and Intelligent Computing in University of Shandong (Linyi University), and the Applied Mathematics Enhancement Program of Linyi University.

\section{References}

[1] S. I. Resnick, Extreme Values, Regular Variation, and Point Processes, Springer-Verlag, New York, NY, USA, 1987.

[2] E. Seneta, "Regularly varying functions," in Lecture Notes in Math, vol. 508, Springer-Verlag, Berlin, Germany, 1976.

[3] S. Kong, M. Saif, and B. Liu, "Observer design for a class of nonlinear fractional-order systems with unknown input," Journal of the Franklin Institute. Engineering and Applied Mathematics, vol. 354, no. 13, pp. 5503-5518, 2017.

[4] L. Mi and B. Liu, "Second order expansion for blowup solutions of semilinear elliptic problems," Nonlinear Analysis. Theory, Methods \& Applications, vol. 75, no. 4, pp. 2591-2613, 2012.

[5] L. Mi and B. Liu, "The second order estimate for the solution to a singular elliptic boundary value problem," Applicable Analysis and Discrete Mathematics, vol. 6, no. 2, pp. 194-213, 2012. 
[6] L. Mi, Y. Qi, and B. Liu, "Blow-up rate of the unique solution for a class of one-dimensional p-Laplacian equations," Nonlinear Analysis. Real World Applications, vol. 13, no. 6, pp. 2734-2746, 2012.

[7] Z. Zhang, "Boundary blow-up elliptic problems with nonlinear gradient terms," Journal of Differential Equations, vol. 228, no. 2, pp. 661-684, 2006.

[8] E. Nakai, "Hardy-Littlewood maximal operator, singular integral operators and the Riesz potentials on generalized Morrey spaces," Mathematische Nachrichten, vol. 166, pp. 95-103, 1994.

[9] J. Morrey, "On the solutions of quasi-linear elliptic partial differential equations," Transactions of the American Mathematical Society, vol. 43, no. 1, pp. 126-166, 1938.

[10] B. Muckenhoupt, "Weighted norm inequalities for the Hardy maximal function," Transactions of the American Mathematical Society, vol. 165, pp. 207-226, 1972.

[11] F. J. Martín-Reyes, P. Ortega, and A. de la Torre, "Weights for one-sided operators," in Recent developments in real and harmonic analysis, Applied and Numerical Harmonic Analysis, pp. 97-132, Birkhäuser Boston, Inc., Boston, Mass, USA, 2010.

[12] E. Sawyer, "Weighted inequalities for the one-sided HardyLittlewood maximal functions," Transactions of the American Mathematical Society, vol. 297, no. 1, pp. 53-61, 1986.

[13] H. Aimar, L. Forzani, and F. J. Martín-Reyes, "On weighted inequalities for singular integrals," Proceedings of the American Mathematical Society, vol. 125, no. 7, pp. 2057-2064, 1997.

[14] K. F. Andersen and E. T. Sawyer, "Weighted norm inequalities for the Riemann-Liouville and Weyl fractional integral operators," Transactions of the American Mathematical Society, vol. 308, no. 2, pp. 547-558, 1988.

[15] Z. Fu, S. Lu, Y. Pan, and S. Shi, "Boundedness of one-sided oscillatory integral operators on weighted Lebesgue spaces," Abstract and Applied Analysis, vol. 2014, Article ID 291397, 7 pages, 2014.

[16] Z. Fu, S. Lu, Y. Pan, and S. Shi, "Some one-sided estimates for oscillatory singular integrals," Nonlinear Analysis. Theory, Methods \& Applications, vol. 108, pp. 144-160, 2014.

[17] Z. Fu, S. Lu, S. Sato, and S. Shi, "On weighted weak type norm inequalities for one-sided oscillatory singular integrals," Studia Mathematica, vol. 207, no. 2, pp. 137-151, 2011.

[18] F. Liu and S. Mao, "On the regularity of the one-sided HardyLittlewood maximal functions," Czechoslovak Mathematical Journal, vol. 67(142), no. 1, pp. 219-234, 2017.

[19] F. J. Martín-Reyes, P. Ortega Salvador, and A. de la Torre, "Weighted inequalities for one-sided maximal functions," Transactions of the American Mathematical Society, vol. 319, no. 2, pp. 517-534, 1990.

[20] J. Garcia-Cuerva and J. L. R. De Francia, "Preface," NorthHolland Mathematics Studies, vol. 116, pp. vii-viii, 1985.

[21] L. de Rosa, "Two weight norm inequalities for fractional onesided maximal and integral operators," Commentationes Mathematicae Universitatis Carolinae, vol. 47, no. 1, pp. 35-46, 2006. 


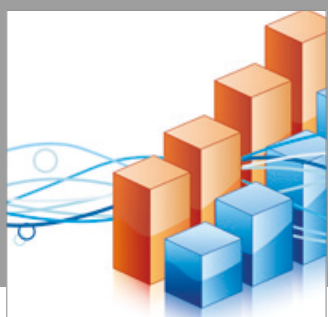

Advances in

Operations Research

vatersals

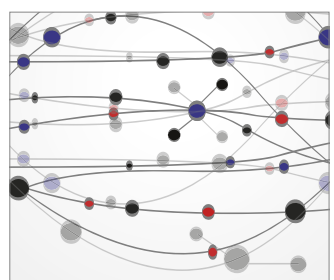

\section{The Scientific} World Journal
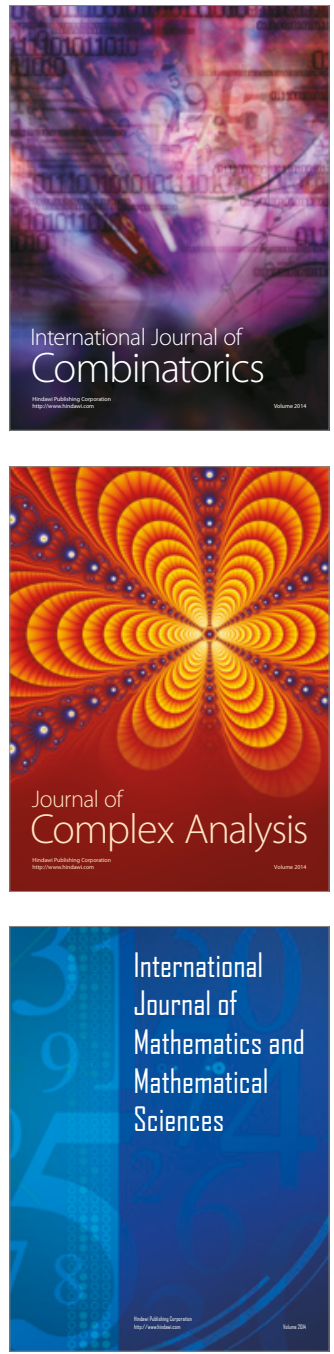
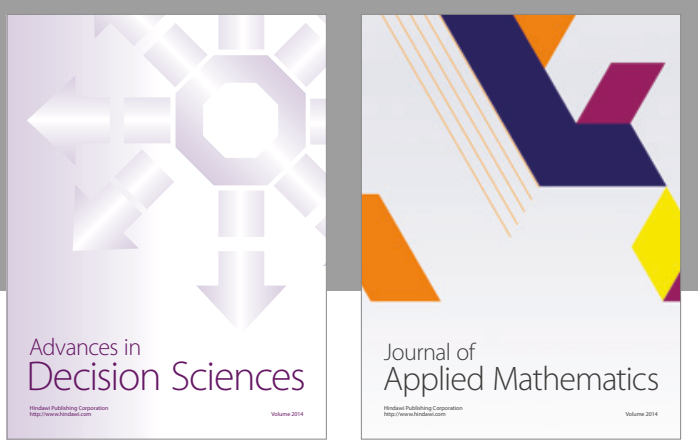

Algebra

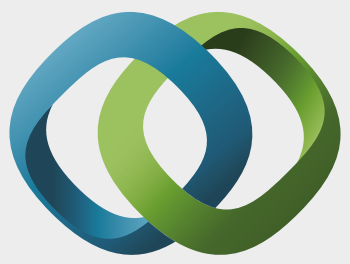

\section{Hindawi}

Submit your manuscripts at

https://www.hindawi.com
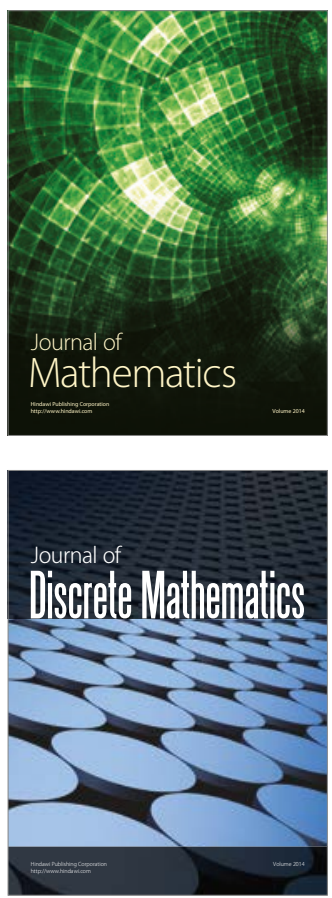

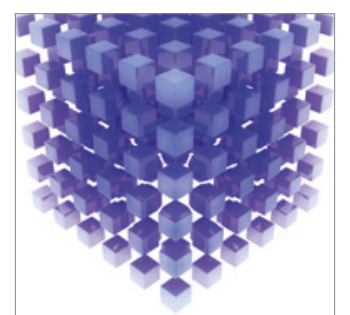

Mathematical Problems in Engineering
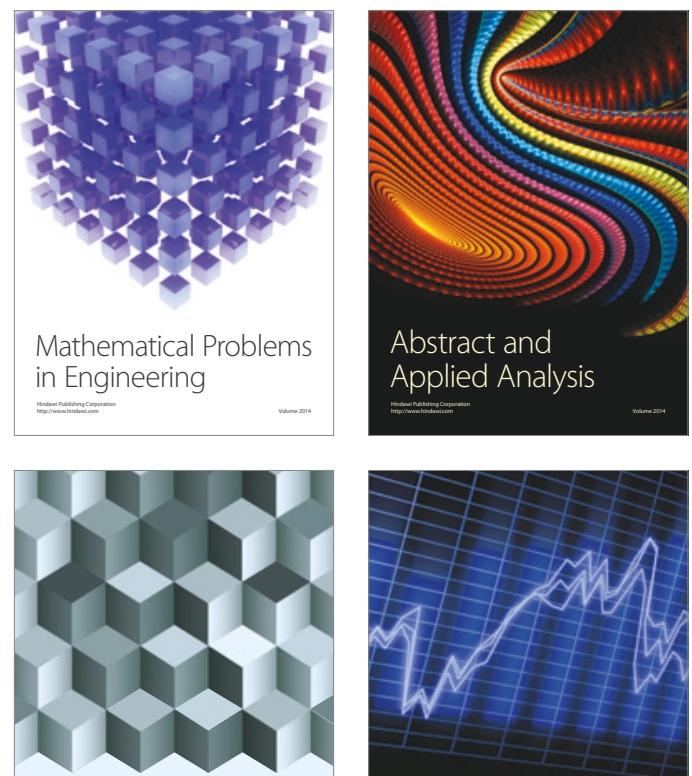

Journal of

Function Spaces

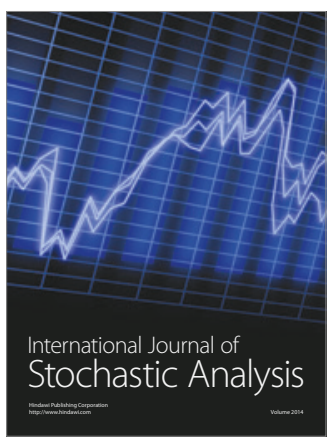

Probability and Statistics
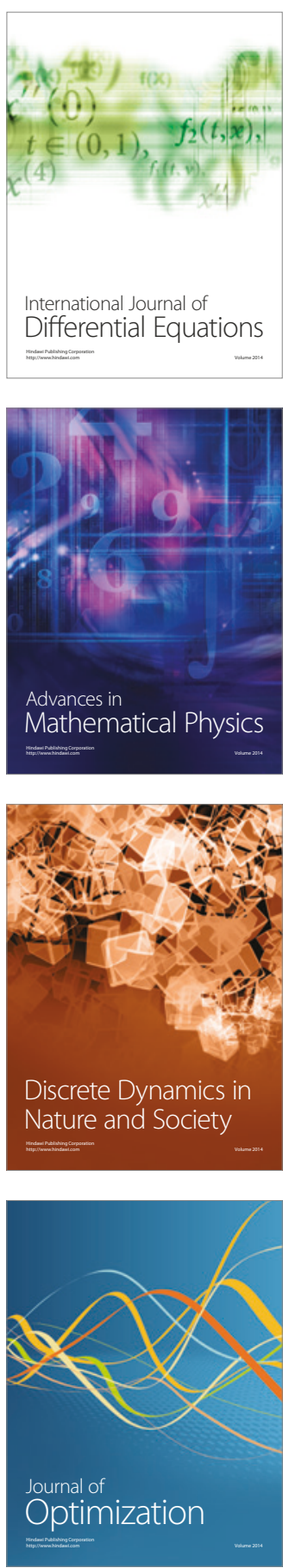